\title{
Analysis of Current Variation Through a Field Effect Transistor Based on Asymmetric Zigzag Carbon Nanotubes
}

Golshad Kheiri ( $\sim$ g.kheiri@urmia.ac.ir )

Urmia university

\section{Research Article}

Keywords: Carbon Nanotube, Field Effect Transistor, Quantum Wire, Zigzag Nanotubes

Posted Date: January 25th, 2022

DOI: https://doi.org/10.21203/rs.3.rs-1057396/v1

License: (c) (1) This work is licensed under a Creative Commons Attribution 4.0 International License.

Read Full License 


\title{
Analysis of current variation through a field effect transistor based on asymmetric zigzag carbon nanotubes
}

\author{
Golshad Kheiri $^{* 1}$ \\ 1 Physics Department, Faculty of Science, Urmia University, Urmia, Iran \\ Corresponding Author Email: g.kheiri @urmia.ac.ir
}

\begin{abstract}
:
Field effect transistors are a group of transistors in which the current is controlled by an electric field. Due to the fact that in these transistors only one type of charge carrier (free electron or hole) is involved in generating electric current, they can be considered as monopolar transistors as opposed to bipolar transistors (which carry both majority and minority at the same time. Are involved in them). Field effect transistors have source, drain, and gate tripods. Field effect transistors can be considered as a voltage sensitive device whose input impedance is very high (about 1014 ohms) and its output impedance is relatively high. Nanotube-based field effect transistors are one of the most useful devices used in future electronic applications. In this type of transistors, metal electrodes act as springs and wells on both sides, and the carbon nanotube between the two acts as a conduit for the carriers. Due to the special properties of carbon nanotubes and their resemblance to quantum wires, we call it a one-dimensional system that does not allow carriers to disperse and thus transfer along it. It will be ballistic. The main issue in this research is to calculate the current passing through a nanotube and mention its importance.

Keywords: Carbon Nanotube, Field Effect Transistor, Quantum Wire, Zigzag Nanotubes
\end{abstract}

\section{Introduction}

Carbon with atomic number 6 is in the sixth group of the periodic table. This element is the main composition of living things. This element has long been known to humans as soot and charcoal. There are other different types of carbon, the only difference being the formation of carbon atoms relative to each other or their lattice structure. Incomplete combustion of many hydrocarbons or organic matter (such as wood or plastic) leaves a black substance called amorphous carbon. This material, which is a waste fuel of organic matter, has long been used to produce human energy. [1-4]. In 1985, Richard Smiley discovered a new carbon structure called Floren. It was the first fluorine to be discovered. The molecule is like a spherical soccer ball and contains 60 carbon atoms at the corners of regular hexagons and a definite number of pentagons. The surface of a sphere cannot be covered by regular hexagons alone, so carbon atoms have to form pentagons in some places to be on a spherical surface. The molecule consists of a structure with 20 hexagons and 12 pentagons [8]. After the report of the discovery of molecules, many scientists began new experiments to make new molecules from carbon. Finally, in 1991, Ejima discovered multi-walled carbon nanotubes [9]. Two years after the discovery of multi-walled carbon nanotubes, Ijima et al, succeeded in making single-walled carbon nanotubes $[10,11]$. Carbon nanotubes can be widely used in electrical components due to their interesting electrical properties. These materials can be a good alternative to metals or semiconductors due to their one-dimensional conductivity at the nanoscale. 

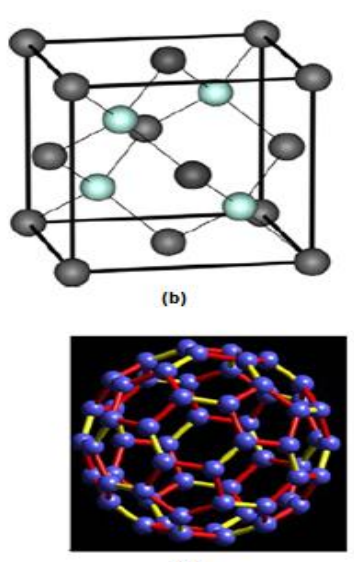

(d)

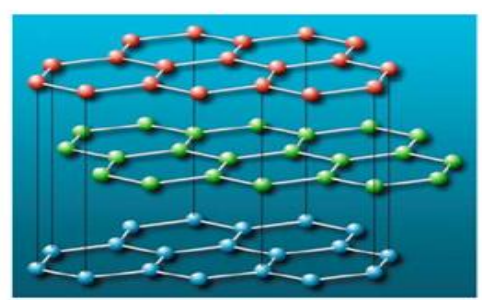

(a)

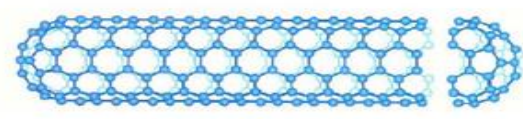

(c)

Figure 1. (a): This figure shows graphite plates. (B): The crystal structure of diamond is shown in this figure. (C) You see an example of an armature nanotube. (D) The molecule, which is a fluorine, is shown in this figure [12].

Depending on their geometric configuration, nanotubes can exhibit conductivity or semiconductor properties, which distinguishes them from other similar materials. In addition to being light, nanotubes are many times stronger than steel [13]. Figure (1) shows the different types of carbon we have introduced here. Chemical vapor deposition, arc, and laser evaporation are the most common methods used to produce nanostructures, including carbon nanotubes. In this method, single-walled or multi-walled nanotubes can be produced. Nanoparticles such as iron, cobalt or nickel nanoparticles alone or in combination are commonly used as catalysts $[14,15]$. The arc method is another method for producing nanotubes. In this method, electrical discharge is performed between two graphite electrodes in a gaseous or liquid medium.

The laser evaporation method was used to produce carbon nanotubes in 1995 by Richard Smelly Group [18]. In this method, a laser is used to evaporate the graphite in the heat furnace. Hot graphite vapor is cooled rapidly High-density nanotubes are produced by the condensation of graphite vapor on the coolant.

Carbon nanotubes have many uses. They can be used as hydrogen storage [19]. They can also be used in electrical devices for nano-sized communication wires. But one of the other applications of nanotubes that we explored in this project is their use in field effect transistors. In Section 2, we examine the structure of carbon nanotube transistors and the connections governing these types of structures. In Section 3, we examine the electric current in semiconductor zigzag nanotubes and examine their electrical resistance. Fifth, conclusions and references are given in order.

\section{Investigating the structure of nanotube carbon transistors}

As shown in Figure 1, a metal-oxide-semiconductor transistor consists of three metal bases called springs, gates, and doors, and a substation of semiconductors with specific impurities. An insulator separates the semiconductor bed and the metal bases. As you can see in Figure 1, a small area of the bottom of the door and the well is made by semiconductor with anti-bed contamination so that there is a distance between the two and this distance is filled by the same bed material. When no voltage is applied to the port, the two back-to-back joints between the door and the spring prevent current from flowing. When voltage is applied to the gate, an electric charge is induced at the junction of the semiconductor and the insulation. These induced charges create a conduit between the source and the reaper. The amount of this conductivity increases with increasing voltage. However, as the transistor shrinks, the thickness of the oxide and, of course, the length of the conductor decreases. The idea of using carbon nanotubes as a channel instead of a semiconductor with a specific contamination has been pursued by many scientists [20, 21]. So that theoretical calculations and laboratory observations have shown that these materials are a good alternative to new transistors due to their one-dimensional strip structure. A transistor that uses a carbon nanotube as a conduit for current is called a carbon nanotube field effect transistor (Figure 2). 


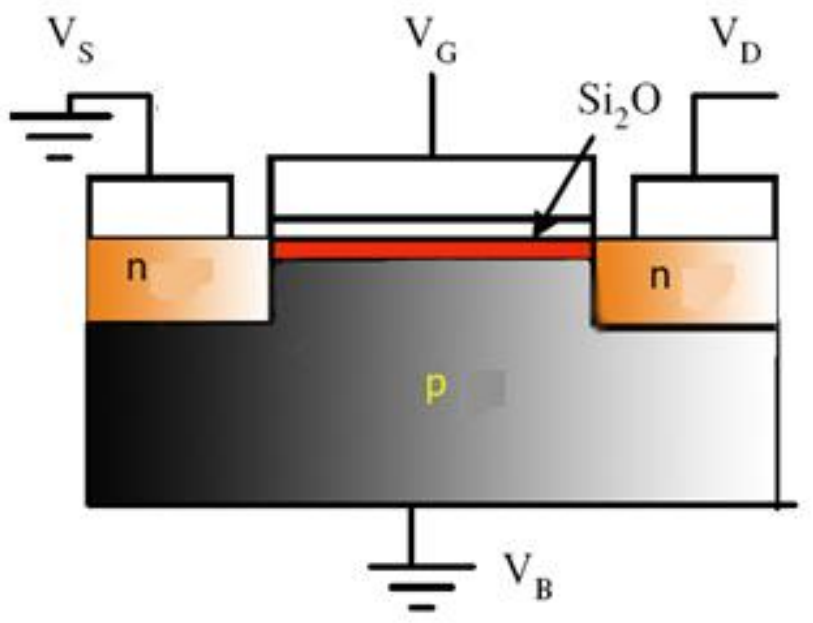

Figure 1. In this figure a schematic of a metal-oxide-semiconductor transistor

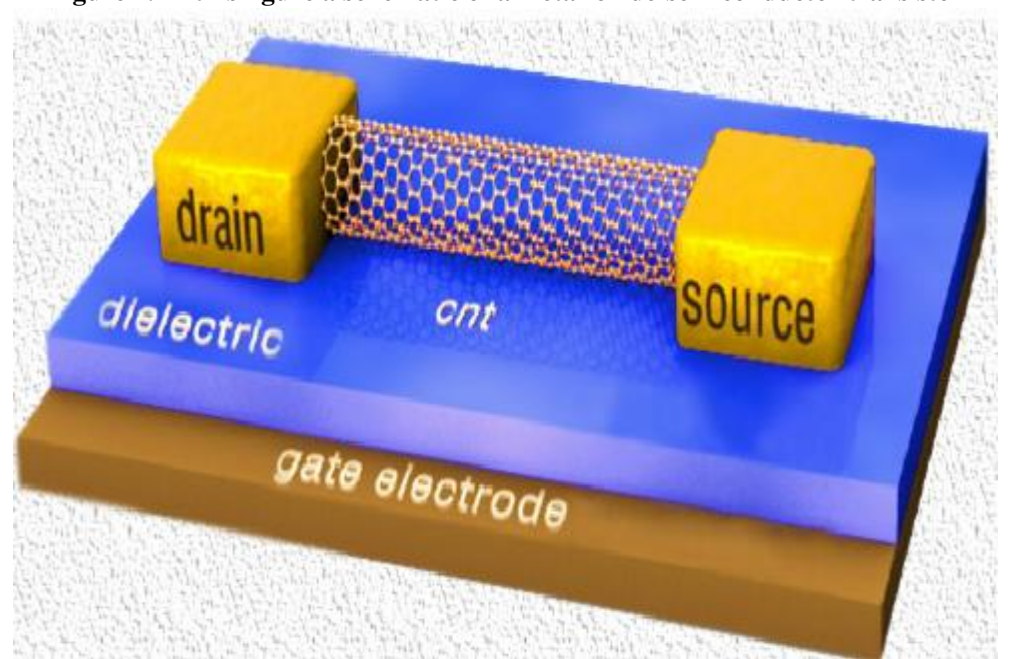

Figure 2. Schematic of a transistor of a nanotube carbon. As shown, we place a nanotube between the source and the door as a conduit.

\subsection{Energy levels of carbon nanotubes}

A carbon nanotube is considered to be a graphite plate wrapped around a cylinder. The number of lines allowed in the first Brillouin region of a single nanotube cell is equal. Since the component of the wave vector parallel to the axis of the infinite nanotube is somewhat continuous, so it can be considered as a continuous vector in the $\vec{K}_{\|}$direction. In the general case, the total wave vector of the nanotube is determined as the sum of the vertical components parallel to the axis of the nanotube.

$$
\overrightarrow{\mathrm{K}}_{\mathrm{NT}}^{v}=\left(\mathrm{k} \frac{\overrightarrow{\mathrm{K}}_{\|}}{\left|\overrightarrow{\mathrm{K}}_{\|}\right|}+v \overrightarrow{\mathrm{K}}_{\perp}\right), \quad \frac{\pi}{|\overrightarrow{\mathrm{T}}|} \leq \mathrm{k} \leq \frac{\pi}{|\overrightarrow{\mathrm{T}}|}, \quad v=01, \ldots, \mathrm{N}-1
$$

Because a one-dimensional network with a constant lattice $\mathrm{T}$ has an inverse network equal to the range of the first Brillouin region $-\frac{\pi}{\mathbf{T}} \leq \mathrm{k} \leq \frac{\pi}{\mathbf{T}}$ [22-19]. Therefore, in relation (1), it changes between the mentioned range. The curves obtained on the graphite energy levels per equation (1) are the same as the nanotube energy levels. Therefore, the energy levels of carbon nanotubes are obtained as follows. 
$\mathrm{E}_{\mathrm{NT}}^{v}(\mathrm{k})=\mathrm{E}_{\text {graphit }}\left(\mathrm{k} \frac{\overrightarrow{\mathrm{K}}_{\|}}{\left|\overrightarrow{\mathrm{K}}_{\|}\right|}+v \overrightarrow{\mathrm{K}}_{\perp}\right)$

Figure 3 shows these energy levels for zigzag and armature nanotubes. As you can see in this diagram, although the number of lines allowed should be equal to 20 for nanotubes $(10,10)$ and $(10,0)$, but only 11 modes can be seen for the conduction band and capacity. This is due to the permissiveness of the energy lines allowed in zigzag and armature nanotubes. As you can see in Figure ( $3 \mathrm{a}$ ), the capacitance strip does not touch the conduction band in zigzag nanotubes $(10,0)$ and there is a small energy gap between them. [14] Therefore, the nanotube $(10,0)$ is semiconductor. But in the case of nanotubes, the armature has touched the capacity of the conducting tape. Therefore, nanotubes $(10,10)$ show their conductive properties, as we will see later, all armature nanotubes are conductive. As you can see in Figure 3, the energy levels of the graphite plate at the $\mathrm{K}$ points in the first Brillouin region are stagnant. Therefore, if the carbon nanotube wave vector passes through these points, the resulting nanotube will show conductive properties, otherwise the nanotube will show semiconductor properties. The position of the point $K$ is given by $\vec{K}=\left(\frac{2 \pi}{\sqrt{3} a}, \frac{2 \pi}{3 a}\right)$ the vector drawn from the origin in the first Brillouin region. According to Equations (3), the following equation can be extracted. $\overrightarrow{\mathrm{K}}_{\mathrm{NT}}^{v} \cdot \overrightarrow{\mathrm{C}}_{\mathrm{h}}=2 \pi v$

The chiral vector is defined as follows

$\overrightarrow{\mathrm{C}}_{\mathrm{h}}=\left(\frac{(\mathrm{n}+\mathrm{m}) \sqrt{3}}{2}, \frac{\mathrm{n}-\mathrm{m}}{2}\right) \mathrm{a}$

If we place the nanotube wave vector in equation (5) equal to $\vec{K}=\left(\frac{2 \pi}{\sqrt{3} a}, \frac{2 \pi}{3 a}\right)$, we get the following equation. Since the numbers $\mathrm{n}, \mathrm{m}$, and all are integers, the condition for the passage of a carbon nanotube wave vector according to Equation (6) is that the integer is 3 . This condition is equivalent to saying that is a multiple of 3 . Therefore, if condition (6) is met, we have a conductive nanotube.
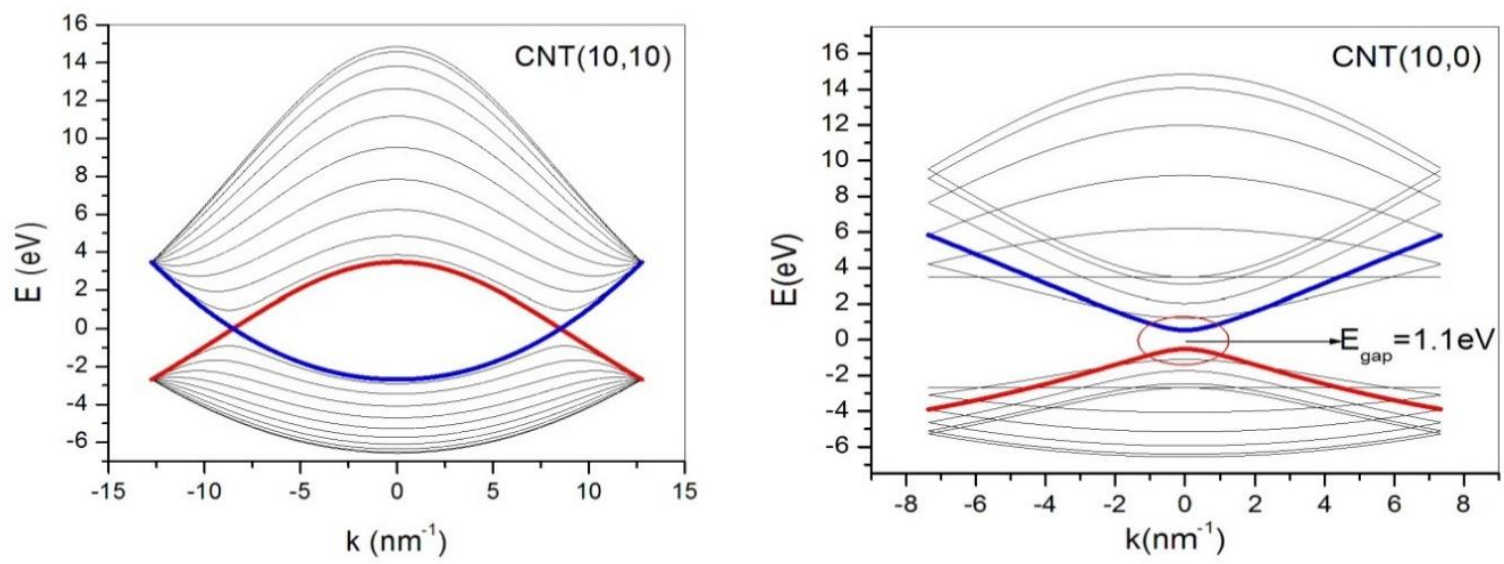

Figure 3. (a) Graph of energy levels for zigzag nanotubes (10,0) (b) Energy levels of armature nanotubes $(10,10)$. 


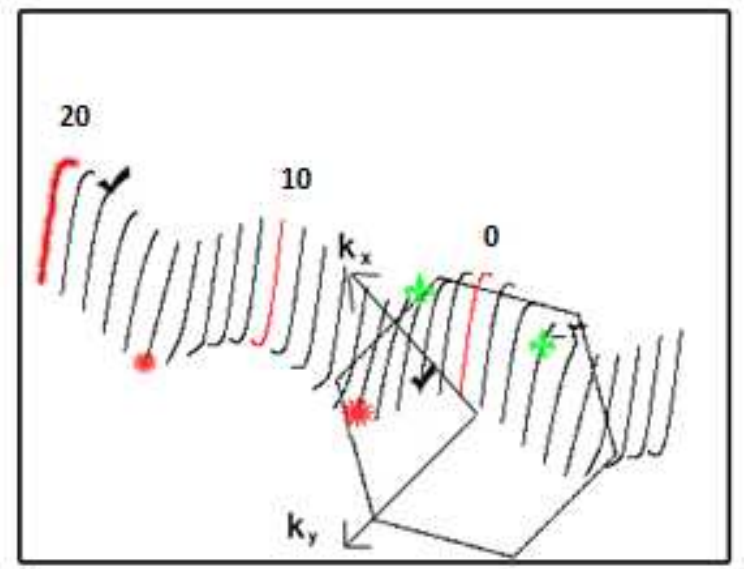

Figure 4. In this figure, we have shown the permissible energy lines for nanotubes (10,0). Although the number of permissible lines is 20 , the lines that go beyond have an equivalent of 0 . We have shown these equivalent lines with special symbols. Also, for quantum numbers that indicate energy levels that are negative, there are equivalent lines between 0 and this will be used many times in the next chapter to save the number of calculations.

Electrons are fermions and follow Fermi-Dirac statistics. Dirac form distribution function is expressed as follows.

$$
f(\varepsilon)=\frac{1}{1+e^{\frac{\varepsilon-\mu}{K_{B} T}}}
$$

In this regard, $\varepsilon$ is the electron energy and $\mu$ is the chemical potential of the system. At zero temperature the chemical potential is the same as the Fermi energy of the particles. Since $\mathrm{K}_{\mathrm{B}} \mathrm{T}$ is $0.01 \mathrm{eV}$ at room temperature, the distribution function Eqe. 7 changes only around $\mu=\varepsilon$. The chemical potential up to the second order correction of temperature for metals is expressed as follows [27].

$$
\mu=\varepsilon_{F}-\frac{\pi^{2}}{6}\left(k_{B} T\right)^{2} \frac{\operatorname{DOS}^{\prime}\left(\varepsilon_{F}\right)}{\operatorname{DOS}\left(\varepsilon_{F}\right)}
$$

In this regard, DOS is the state density. From this relation it can be quickly understood that where the derivative of the density of states relative to energy is zero, the chemical potential is equal to the Fermi energy. But the distribution function changes in the scattering, that is, the electric field or collisions change the nature of the distribution function. In such cases, instead of the distribution function (7), they introduce an unbalanced function called g, which expresses the nature of the distribution function in different distributions. The unequal distribution function $\mathrm{g}$ is generally expressed as the Boltzmann equation [27].

$$
\frac{\partial \mathrm{g}}{\partial \mathrm{t}}+\overrightarrow{\mathrm{v}} \cdot \frac{\partial}{\partial \overrightarrow{\mathrm{r}}} \mathrm{g}+\overrightarrow{\mathrm{F}} \cdot \frac{\partial}{\partial \overrightarrow{\mathrm{k}}} \mathrm{g}=\sum_{\overrightarrow{\mathrm{k}}^{\prime}} \frac{\mathrm{d} \overrightarrow{\mathrm{k}}}{8 \pi^{3}}\left\{\mathrm{~W}_{\overrightarrow{\mathrm{k}}, \overrightarrow{\mathrm{k}}^{\prime}} \mathrm{g}(\overrightarrow{\mathrm{k}})\left[1-\mathrm{g}\left(\overrightarrow{\mathrm{k}}^{\prime}\right)\right]-\mathrm{W}_{\overrightarrow{\mathrm{k}}^{\prime}, \mathrm{k}} \mathrm{g}\left(\overrightarrow{\mathrm{k}}^{\prime}\right)[1-\mathrm{g}(\overrightarrow{\mathrm{k}})]\right\}
$$

Its time, which gives a measure of the probability of an electron colliding in a given period of time, is equal to the inverse of the total scattering rate from a state $\vec{k}$ to all possible $\overrightarrow{k^{\prime}}$ states [30, 31].

$$
\frac{1}{\tau(\overrightarrow{\mathrm{k}})}=\sum_{\overrightarrow{\mathrm{k}}^{\prime}} \mathrm{W}_{\overrightarrow{\mathrm{k}}, \overrightarrow{\mathrm{k}}^{\prime}}
$$

From quantum mechanics we know that the rate of scattering from one state to another is expressed by the Fermi golden rule as follows.

$$
W_{i \rightarrow f}=\frac{2 \pi}{\hbar}|\langle f|H| i\rangle|^{2} \delta\left(E_{i}-E_{f} \pm \hbar \omega\right)
$$


In this regard, Hamiltonian $\mathrm{H}$ is an interaction that causes scattering. As mentioned earlier, in the absence of impurities and crystalline defects, phonons are dispersing agents in solids. The electron-phonon scattering rate in carbon nanotubes is given by the following equation $[32,33]$.

$$
\mathrm{W}_{\overrightarrow{\mathrm{k}}, \overrightarrow{\mathrm{k}}^{\prime}}=\sum_{\text {all }, \mu} \frac{\hbar \mathrm{D}^{2} \operatorname{DOS}(\overrightarrow{\mathrm{k}}+\overrightarrow{\mathrm{q}})}{\rho \mathrm{d} \mathrm{E}_{\mathrm{p}}(\overrightarrow{\mathrm{q}}, \mu)}\left[\mathrm{N}_{\mathrm{p}}+\frac{1}{2} \pm \frac{1}{2}\right]
$$

The phonon energy in a carbon nanotube is given by Equation (13). The Bose-Einstein occupation number is expressed as follows.

$$
N_{p}=\frac{1}{e^{\frac{E_{p}}{k_{B} T}}-1}
$$

Once (13) is obtained, it can be placed in Equation (7) to obtain the unequal distribution function g. Equation (14) is a nonlinear differential equation, but it can be converted to a linear equation by approximating its time. The approximate time and place are expressed as follows.

$\frac{\partial}{\partial \mathrm{t}} \mathrm{g}(\overrightarrow{\mathrm{r}}, \overrightarrow{\mathrm{k}}, \mathrm{t})+\overrightarrow{\mathrm{v}} \cdot \frac{\partial}{\partial \overrightarrow{\mathrm{r}}} \mathrm{g}(\overrightarrow{\mathrm{r}}, \overrightarrow{\mathrm{k}}, \mathrm{t})+\overrightarrow{\mathrm{F}} \cdot \frac{\partial}{\partial \overrightarrow{\mathrm{k}}} \mathrm{g}(\overrightarrow{\mathrm{r}}, \overrightarrow{\mathrm{k}}, \mathrm{t})=-\frac{\mathrm{g}(\overrightarrow{\mathrm{r}}, \overrightarrow{\mathrm{k}}, \mathrm{t})-\mathrm{g}^{\circ}(\overrightarrow{\mathrm{r}}, \overrightarrow{\mathrm{k}}, \mathrm{t})}{\tau(\overrightarrow{\mathrm{k}})}$

When the distribution function is obtained, the expected value of each physical quantity is obtained as follows.

$$
\bar{A}(t)=\sum_{n, \vec{k}} A(\vec{k}, t) g_{n}(\vec{k}, t)
$$

The approximation of time and family obviously means that time and family are independent of scattering. The go function is a locally balanced distribution function that is independent of applied fields and scattering. In fact, this function is the same as the Fermi-Dirac distribution function for fermions. By solving Equation (14), the distribution function in a homogeneous space environment in which the spatial derivatives are zero is given as follows [27].

$g_{n}(\vec{k}, t)=g_{n}^{\circ}(\vec{k})+e \int_{-\infty}^{t} d t^{\prime} P\left(t, t^{\prime}\right) \frac{\partial}{\partial \varepsilon_{n}\left(k\left(t^{\prime}\right)\right)} g_{n}^{\circ}\left(\vec{k}\left(t^{\prime}\right)\right) \vec{v} \cdot \vec{E}$

In the above relation, $\vec{E}$ is an electric field and it should also be noted that in order to obtain relation (15), semiclassical equations of motion have been used. These equations are expressed as follows.

$$
\begin{aligned}
& \hbar \frac{\mathrm{d}}{\mathrm{dt}} \overrightarrow{\mathrm{k}}=-\mathrm{e} \overrightarrow{\mathrm{E}} \\
& \overrightarrow{\mathrm{v}}=\frac{1}{\hbar} \frac{\partial}{\partial \vec{k}} \varepsilon_{n}(\vec{k})
\end{aligned}
$$

Thus the average velocity of the electrons in the energy band is zero and no net current is generated. This makes conduction in zigzag nanotubes impossible. In order to be able to use such nanotubes to transport carriers, it is necessary to raise the form level, which is normally zero (Figure 2), to the conduction band. In field effect transistors, this is easily possible by placing the nanotube as a conduit between the door and the source (Figure 3). In this situation, the nanotube and the port form a capacitor. By applying a voltage to the port, some charge is injected into the nanotube, which causes the Fermi surface to move. If a positive potential is applied to the port, the Fermi surface will be pulled into the conduction band [34]. The Fermi wave vector is given by the following equation [35].

$$
\begin{aligned}
& \mathrm{k}_{\mathrm{F}}=\frac{\pi \mathrm{Q}}{4 \mathrm{eL}} \\
& \mathrm{Q}=\mathrm{C}\left(\mathrm{V}_{\mathrm{G}}-\mathrm{V}_{\mathrm{Th}}\right)
\end{aligned}
$$

In this connection, $\mathrm{V}_{\text {th }}$ is the threshold potential of the transistor, and $\mathrm{C}$ is the capacitance of the port capacitor and the cylindrical nanotube. The capacitance of the port-nanotube capacitor is introduced as follows $[6,36]$. 


$$
C=\frac{2 \pi \kappa \varepsilon_{0} L}{\ln \left(\frac{2(t+r)}{r}\right)}
$$

In this relation $\mathrm{k}$ is the dielectric constant of the gate oxide, $\mathrm{r}$ is the nanotube radius and $\mathrm{t}$ is the gate oxide thickness. Fermi energy is obtained by obtaining the number of charges injected into the nanotube (Figure 4).

\section{Simulation results}

CNTFET transistors, like MOSFETs, do not have two types and are equivalent to type N or type P based on the bias of their bases. The following are the parameters of a CNT transistor required to simulate electronic circuits.

- Ad equivalent to $\mathrm{W}$ in MOSFETs $(150 \mathrm{~nm})$

- As equivalent to $\mathrm{L}$ in MOSFETs $(45 \mathrm{~nm})$

Dia diameter of nanotube carbon, which in this simulation is equal to 32 nanometers

If necessary, the value of Fermi voltage is also specified. Other parameters that can be defined are:

- Channel length (Lch) which is considered equal to the length of the gate, which is usually equal to $120 \mathrm{~nm}$

- The effective gate length of L_geff, which is usually considered to be $100 \mathrm{~nm}$.

- The thickness of the oxide ( $\mathrm{t} \_\mathrm{ox}$ ) is the default of $15 \mathrm{~nm}$.

- The number of tubes indicated by tubes is 8 by default.

In the simulation, the simulation temperature must be mentioned, which in this research is considered to be equal to 25 degrees Celsius. Figure 5 shows a current-voltage diagram of an n-type CNTFET transistor. As can be seen, with increasing voltage, the current of the CNTFET transistor starts from very small values and increases after 40 volts.

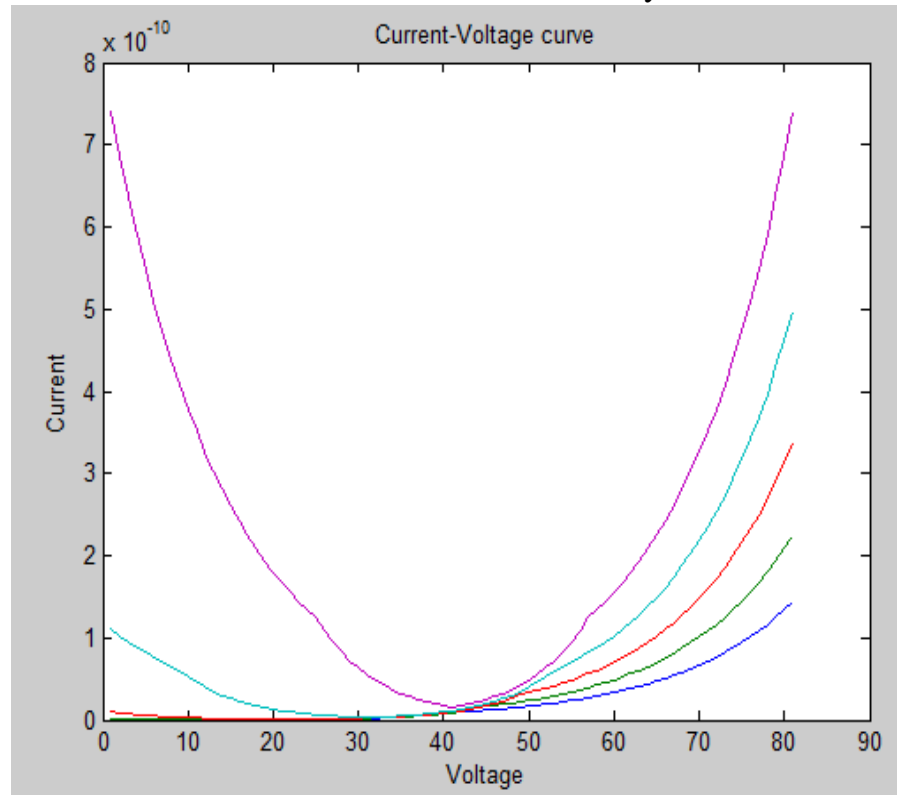

Figure 5 Voltage-current in an NCNTFET In this figure the horizontal axis is the voltage axis (Volt) and the vertical axis is the current axis (Amp). The curves are blue, green, red, and pale blue, respectively. , 0.8, 1.2, 2 .

Figure 6 is the same as Figure 5, which shows this for a PCNTFET transistor. As can be seen, with increasing voltage, the current of the CNTFET transistor starts from very small values and increases after 40 volts. 


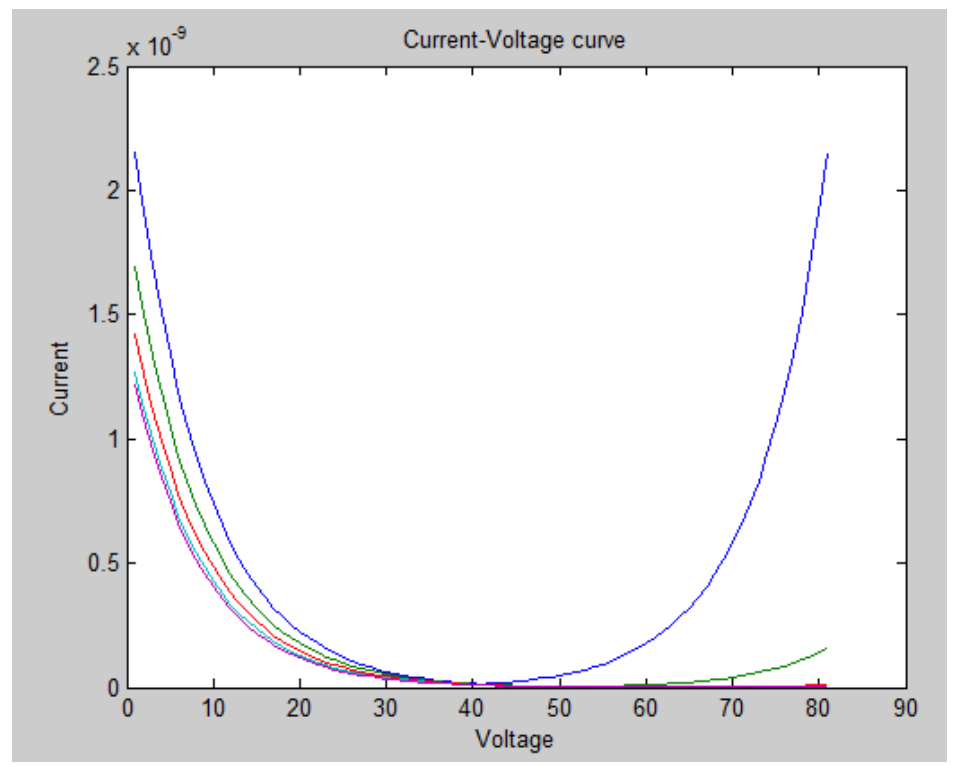

Figure 6 Voltage-current curve for a PCNTFET In this figure the horizontal axis is the voltage axis (Volt) and the vertical axis is the current axis (Amp). The curves are blue, green, red, and pale blue, respectively. 0.6, 0.8, 1.2, 2.

Figure 7 shows the drain-source voltage curves Vds in terms of current in the carbon transistor. Figure 8, as well as Figure 7, shows drain-source voltage curves Vds in terms of current for a PCNTFET. Figure.8 shows the current changes in millivolts of voltage in the source. Figure 9 shows the linear curve of the NCNTFET output voltage in terms of the input voltage with a range of 0.4 volts. As shown in Figure 9, the same diagram for a PCNTFET is shown in Figure 10. Figure 11 shows the CNTFET current changes in terms of Vds voltage changes. Figure 12 shows the Vin curve in terms of Log (Id). Blue, red and green are for voltages Vds $=50,40,30$, respectively. Figure 13 shows the Vin-Log (Id) curve for a PCNTFET. Blue, red and green are for voltages Vds = 50,40,30, respectively.

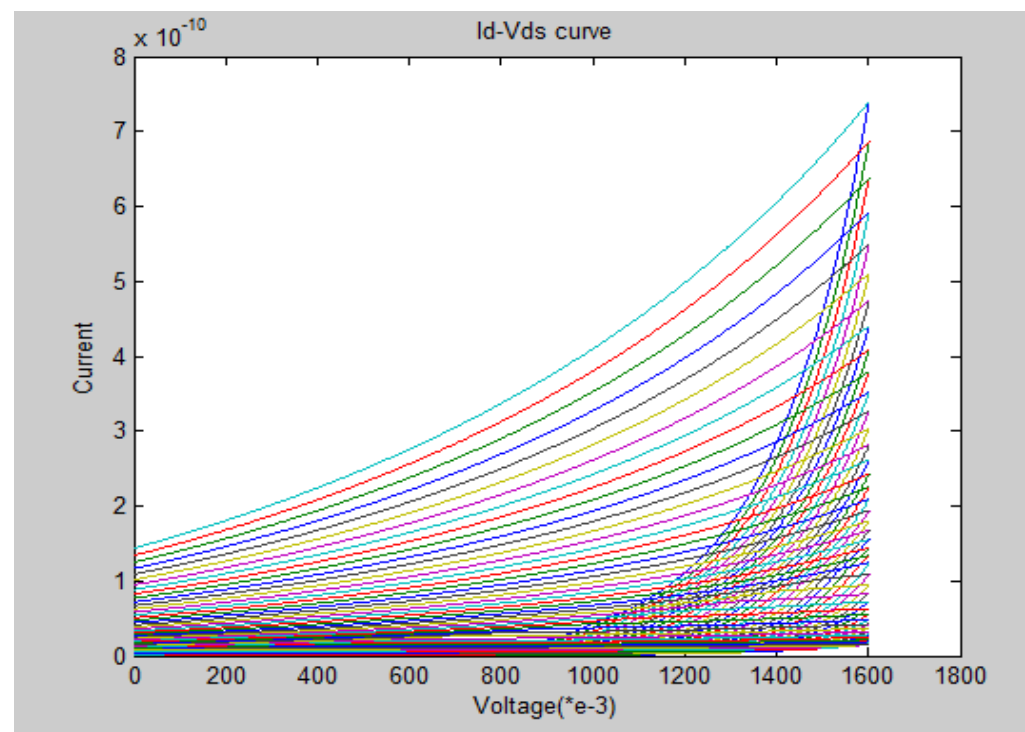

Figure 7 shows the current curve Id (amperes) in Vds (volts) in NCNTFET. 


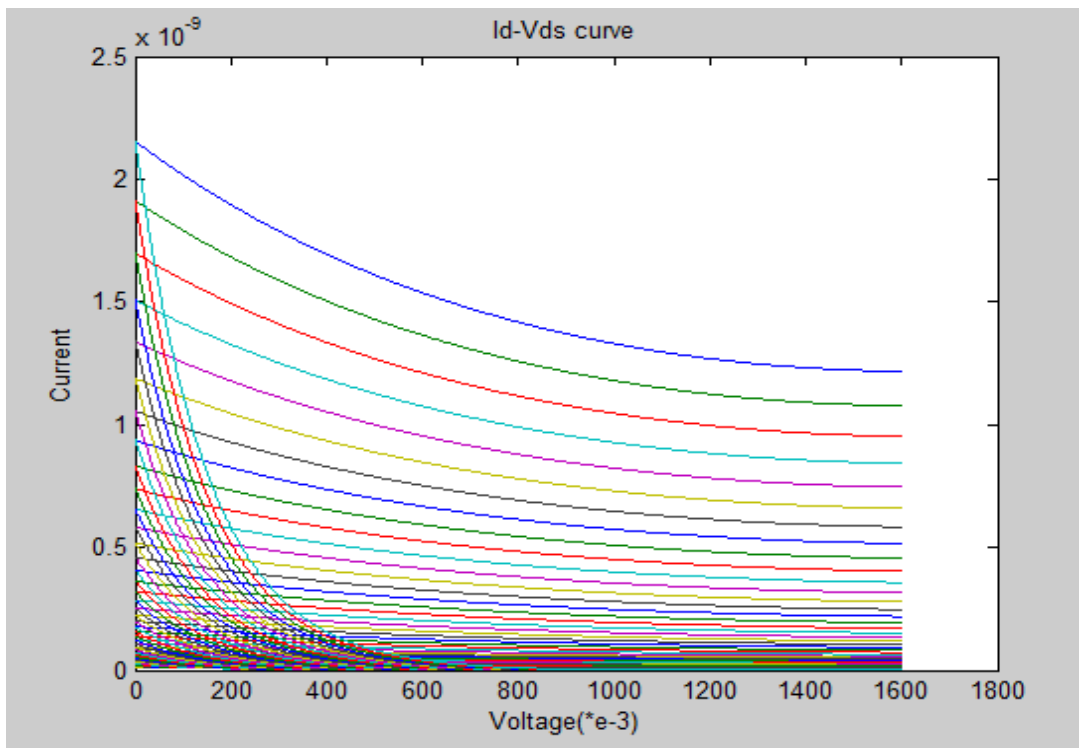

Figure 8 shows the current curve Id (amps) in Vds (volts) in PCNTFET for different Vds voltages.

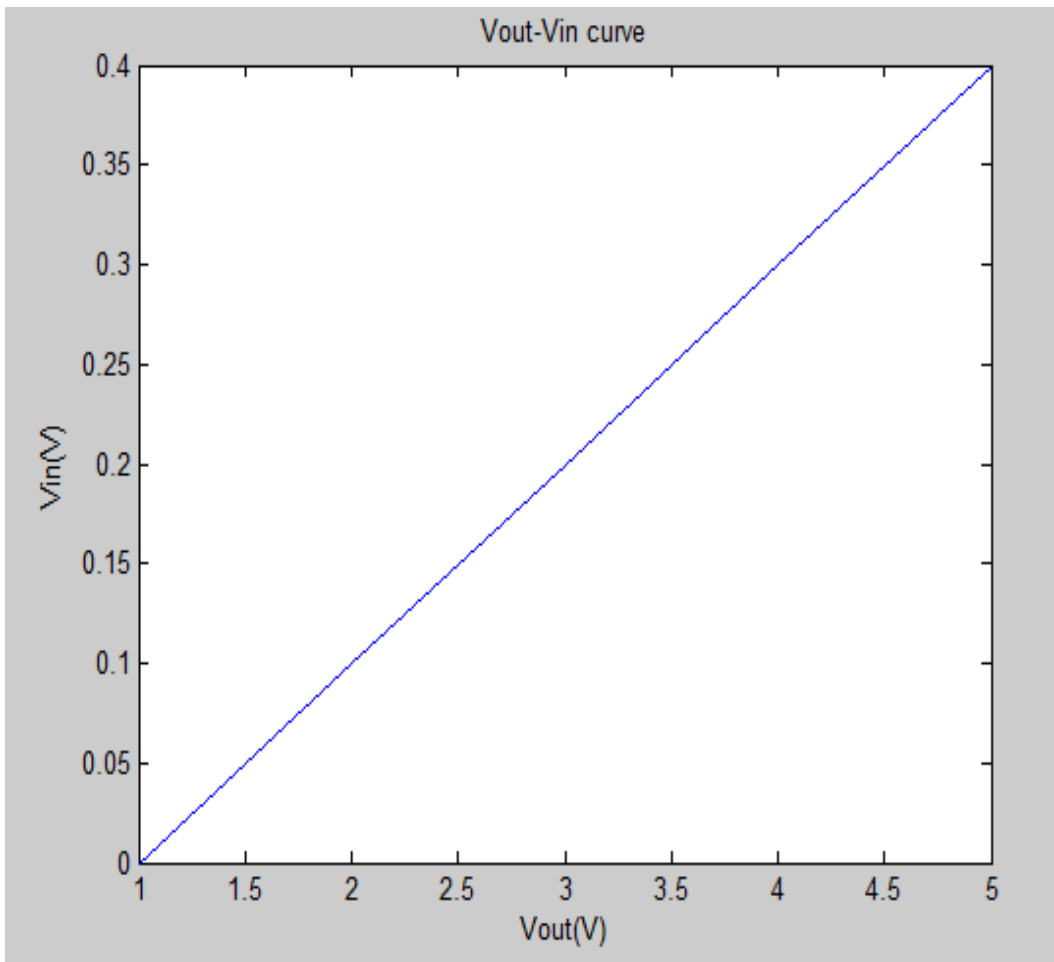

Figure 9 Vout-Vin curve for a NCNTFET 


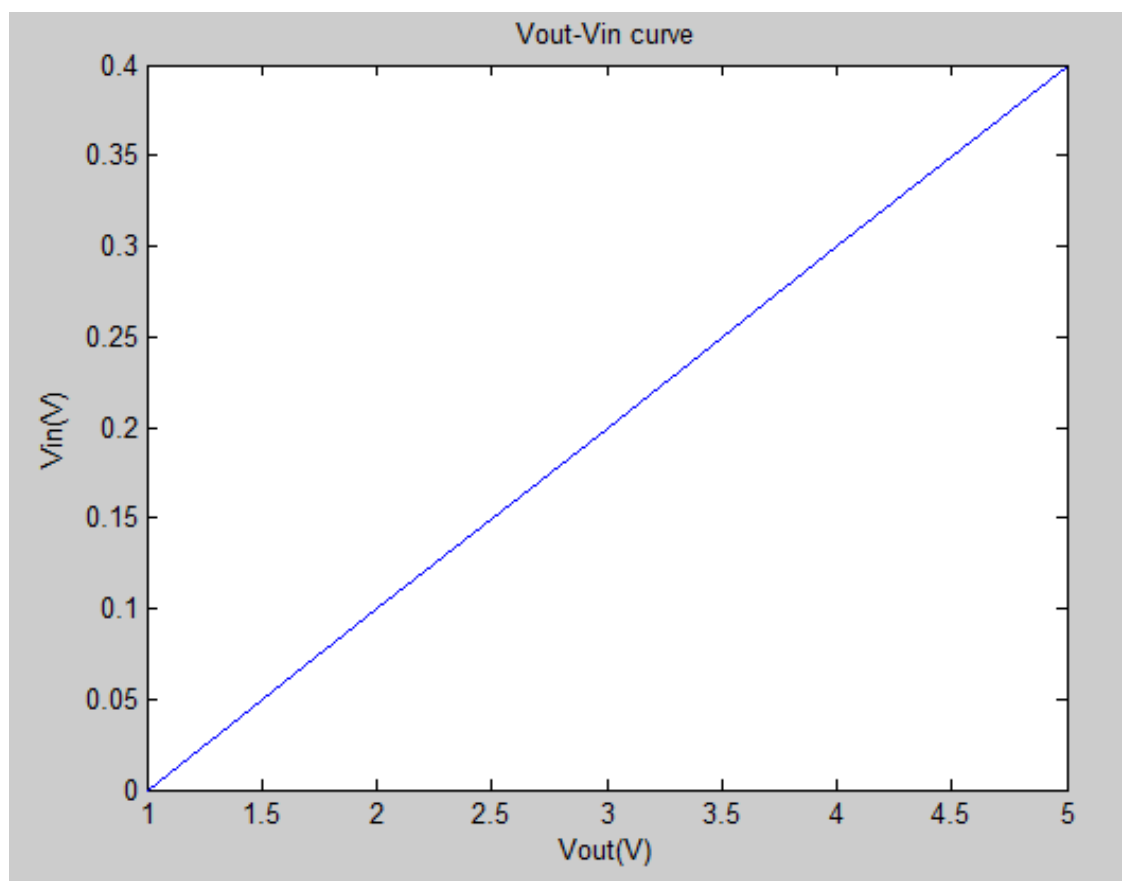

Figure 10 Vout-Vin for a PCNTFET

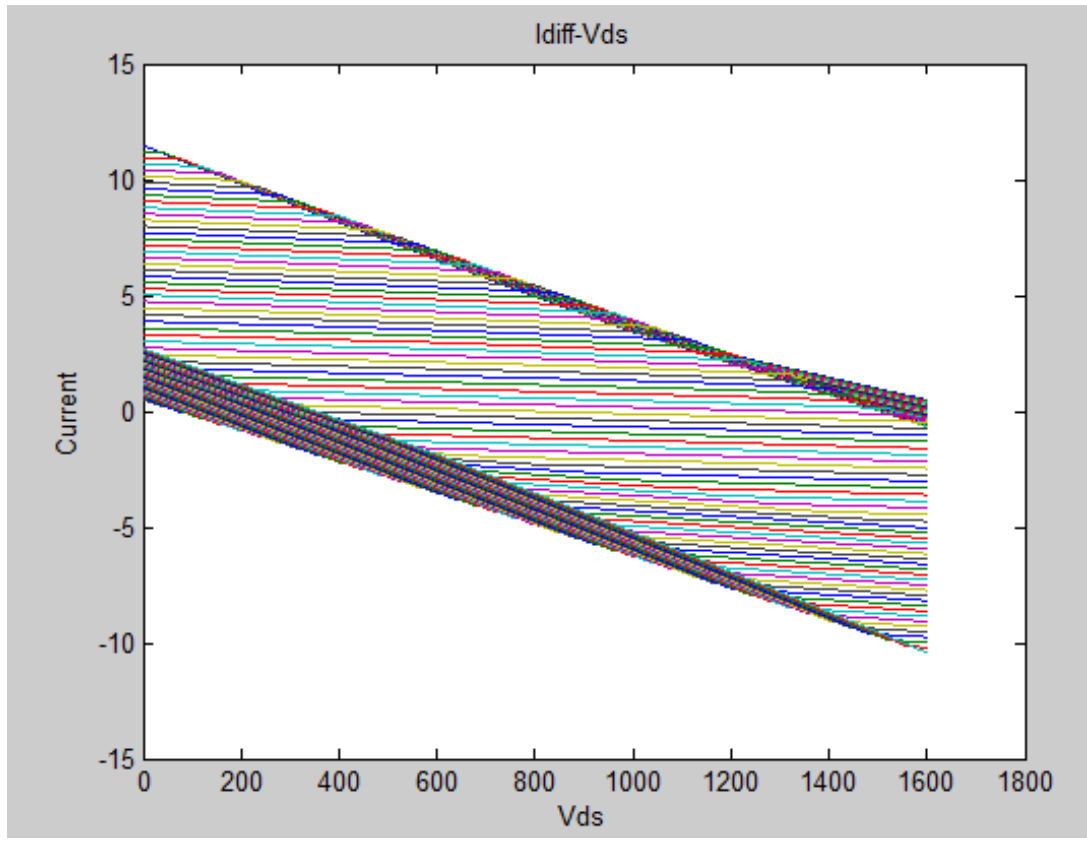

Figure 11 shows CNTFET current changes in terms of Vds voltage changes (each color is different for Vds voltages). 1.6 volts continues The vertical axis is the current axis, the unit of which is nanoamperes 


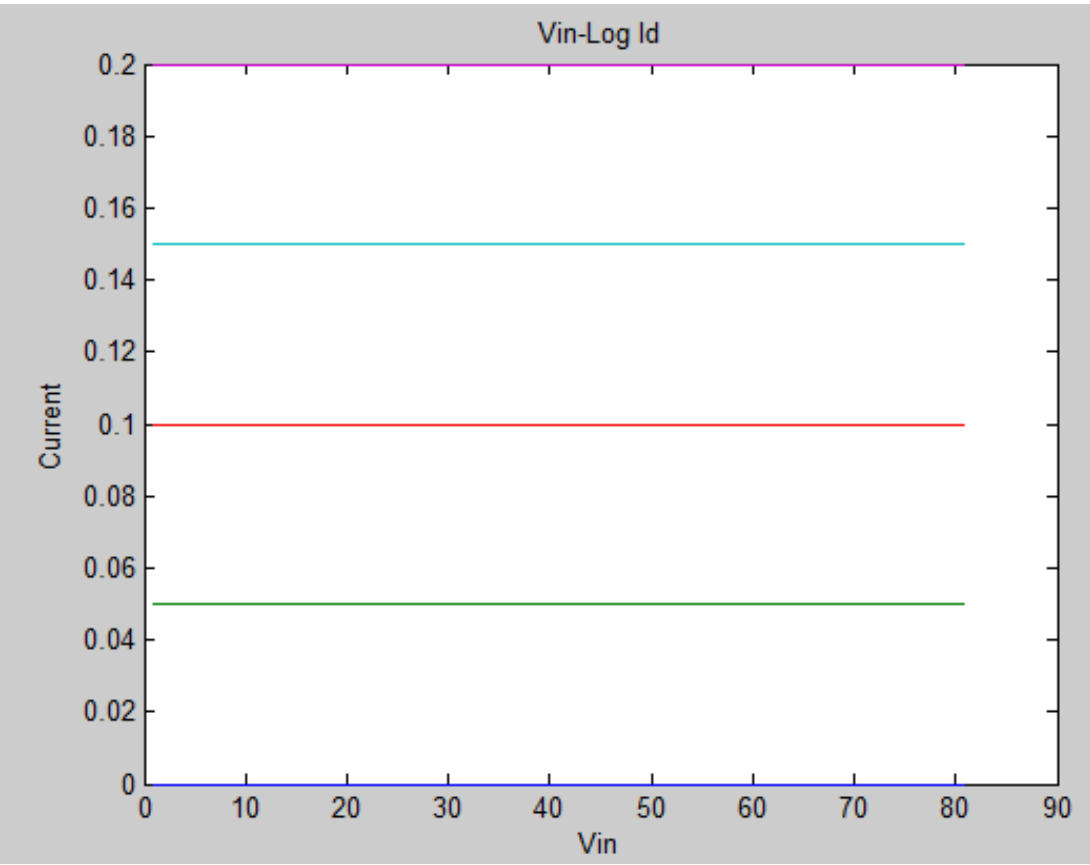

Figure 12 Vin-Log (Id) curve for an NCNTFET is the horizontal voltage axis (volts) and the vertical axis of the current (amps).

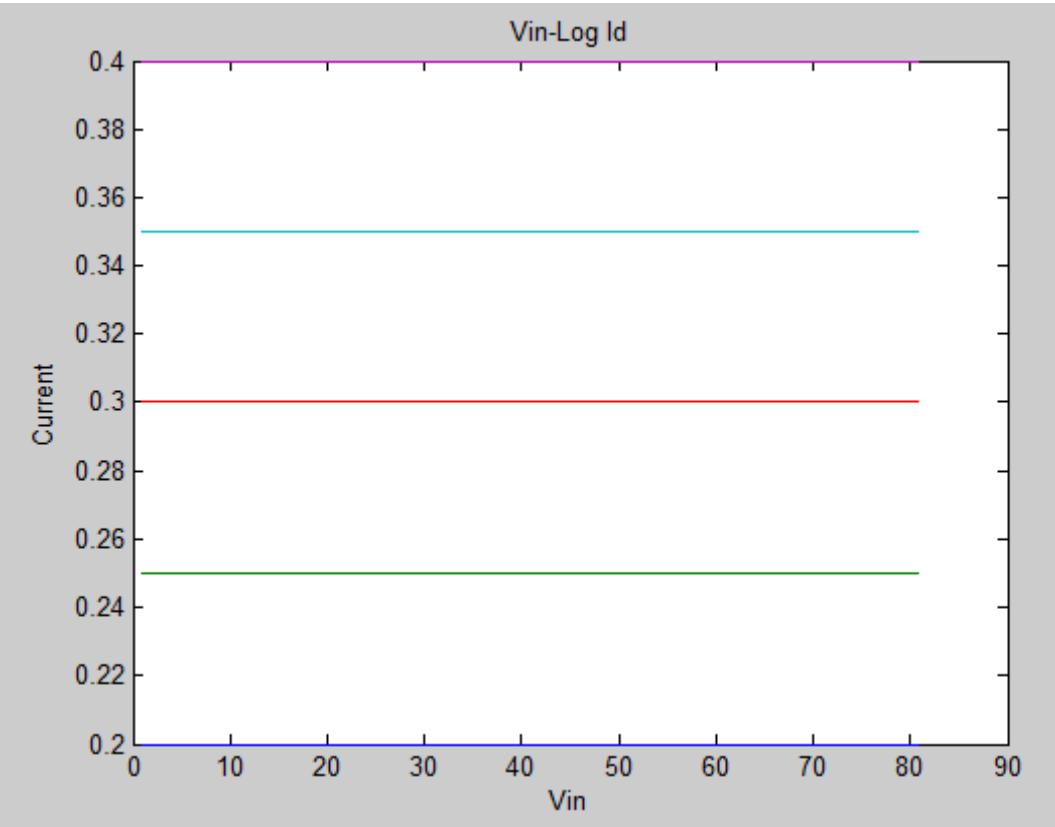

Figure 13 Vin-Log (Id) curve for a PCNTFET is the horizontal axis of voltage (volts) and the vertical axis of current (amps) of each of these catheters are red, green and blue, respectively, with gate voltages of $0.4,0.6$ and 0.8 volts.

\section{Conclusion}

Our calculations show that semiconductor zigzag nanotubes with different diameters at different voltages have maximum mobility, so this should be considered in the design of transistors. Numerous sources also confirm the flow and velocity as we extracted. However, care must be taken to increase (decrease) the current with increasing (decreasing) the diameter of the nanotube, because the study of these materials in the form of quantum wires sometimes leads to a uniform relationship in terms of diameter and current dependence. Another point to consider is the strength of carbon nanotubes. This resistance increases linearly with respect to the potential applied to both, and is not a fixed number. In fact, carbon nanotubes are not ohmic resistors. 


\section{References}

[1] Khademul Islam Majumder, Md. Raied Hasan \& Raquib Ahmed, Thesis on "Improvement of efficiency for solar photovoltaic cell application", Department of Electrical and Electronic Engineering, BRAC University, Dhaka, Bangladesh, April 2010.

[2] De Nicola F, Salvato M, Cirillo C, Crivellari M, Boscardin M, Passacantando M, Nardone M, De Matteis F, Motta N, De Crescenzi M, Castrucci P. $100 \%$ internal quantum efficiency in polychiral single-walled carbon nanotube bulk heterojunction/silicon solar cells. Carbon 2017 30;114:402-10.

[3] 〈http://www.nrel.gov/news/press/2016/25678〉.

[4] Moore Gordon E. Cramming more components onto integrated circuits. Electronics 1965;38(8):1-4.

[5] 〈http://spectrum.ieee.org/tech-talk/computing/hardware/transistors-will-stopshrinking-in-2021-moores-law-roadmap-predicts〉.

[6] Sun Y, He W, Mao Z, Jiao H, Kursun V. High-yield and robust 9T SRAM cell tolerant to removal of metallic carbon nanotubes. IEEE Trans Device Mater Reliab 2017;17(1):20-31.

[7] Beyond CMOS. International Technology Roadmap for Semiconductors 2.0; 1-87; 2015.

[8] Schröter Michael, Claus Martin, Sakalas Paulius, Haferlach M, Wang Dawei. Carbon nanotube FET technology for radio-frequency Electronics: state-of-the-art overview. IEEE J Electron Devices Soc 2013;1(1):9-20.

[9] Marani R, Perri AG. CNTFET-based design of current mirror in comparison with MOS technology. ECS J Solid State Sci Technol 2017;6(5):M60-8. [Jan 1].

[10] Sachid Angada B, Chen Min-Cheng, Hu Chenming. FinFET with high-א spacers for improved drive current. IEEE Electron Device Lett 2016:1-4. http://dx.doi.org/10. 1109/LED.2016.2572664.

[11] Mishra Abhishek, ManishaPattanaik, Sharma Varun Gate Double. Vertical Tunnel FET for Hybrid CMOS-TFET based Low Standby Power Logic Circuits. International Conference on Microelectronics, Communication and Renewable Energy (ICMiCR-; 1-4; 2013.

[12] Chiang Te-Kuang. A new threshold voltage model for short-channel junctionless inverted T-shaped gate FETs (JLITFET). IEEE Trans Nanotechnol 2016;15(3):442-7.

[13] Piccirillo C, Denis CJ, Pullar RC, Binions R, Parkin IP, Darr JA, Castro PM. Aerosol assisted chemical vapour deposition of hydroxyapatiteembedded titanium dioxide composite thin films. J Photochem Photobiol A: Chem 2017;332:45-53. [Jan 1].

[14] Rispal Lorraine, Schwalke Udo. Structural and electrical characterization of carbon nanotube field-effect transistors fabricated by novel selfaligned growth method. In: Proceedings of the 3rd international conference on design and technology of integrated systems in nanoscale era, 2008. DTIS; 2008: 1-5.

[15] Hou B, Wu C, Inoue T, Chiashi S, Xiang R, Maruyama S. Extended alcohol catalytic chemical vapor deposition for efficient growth of singlewalled carbon nanotubes thinner than (6, 5). Carbon 2017;119:502-10. [Aug 31].

[16] Keyn Martin, Schwalke Udo. Multi-CNTFETs for power device applications: Investigation of CCVD grown CNTs by means of atomic force microscopy. In: Proceedings of the 8th international conference on design \& technology of integrated systems in nanoscale era (DTIS); 1 $-5 ; 2013$.

[17] Seichepine F, Rothe J, Dudina A, Hierlemann A, Frey U. Dielectrophoresis-assisted integration of 1024 carbon nanotube sensors into a CMOS microsystem. Adv Mater 2017;29(17). [May 1].

[18] Zhigang Xiao, John Elike, Alishia Reynolds, Roderick Moten, Xiang Zhao, The fabrication of carbon nanotube electronic circuits with dielectrophoresis; 2016. 〈http://dx.doi.org/10.1016/j.mee.2016.07.013〉.

[19] Schroter M, Kolev P, Wang D, Eron M, Lin S, Samarakone N, Bronikowski M, Yu J, Sampat P, Sams P, McKernan S. A 4" wafer photostepperbased carbon nanotube FET technology for RF applications. IEEE MTT-S International Microwave Symposium Digest (MTT). 〈http://dx.doi.org/10.1109/MWSYM.2011.5973204); 1 - 4 .

[20] Taghavi A, Carta C, Meister T, Ellinger F, Claus M, Schroter M. A CNTFET oscillator at 461 MHz. IEEE Microw Wirel Compon Lett 2017. [May 23].

[21] Fedawy Mostafa, Fikry Wael, Alhenawy Adel, Hassan Hazem. I-V characteristics model for ballistic Single Wall Carbon Nanotube Field Effect Transistors (SWCNTFET). IEEE international conference on electronics design, systems and applications (ICEDSA). p. 10-13. $\langle$ http://dx.doi.org/10.1109/ICEDSA.2012.6507775〉: $10-13$.

[22] Tang J, Cao Q, Farmer DB, Tulevski G, Han SJ. High-performance carbon nanotube complementary logic with end-bonded contacts. IEEE Trans Electron Devices 2017;64(6):2744-50.

[23] Lee C-S, Pop E, Franklin AD, Haensch W, Wong H-SP. A compact virtual-source model for carbon nanotube FETs in the sub-10-nm regimePart I: intrinsic elements. IEEE Trans Electron Devices 2015;62(9):3061-9. http://dx.doi.org/10. 1109/TED.2015.2457453.

[24] Janas D, Milowska KZ, Bristowe PD, Koziol KK. Improving the electrical properties of carbon nanotubes with interhalogen compounds. Nanoscale 2017;9(9):3212-21.

[25] Bejenari Igor, Claus Martin. Electron back scattering in CNTFETs. IEEE Trans Electron Devices 2016;63(3):1340-5.

[26] Es-Sakhi AzzedinD, Masud Chowdhury H. Multichannel tunneling carbon nanotube field effect transistor (MT-CNTFET). In: Proceedings of the 27th IEEE international system-on-chip conference (SOCC). p. 156-9. 〈http://dx.doi.org/10.1109/SOCC. 2014.6948918〉.

[27] Lubineau G, Mora A, Han F, Odeh IN, Yaldiz R. A morphological investigation of conductive networks in polymers loaded with carbon nanotubes. Comput Mater Sci 2017;130:21-38. [Apr 1].

[28] Najari Montassar, Frégonèse Sébastien, Maneux Cristell, Mnif Hassène, Masmoudi Nouri, Zimmer Thomas. Schottky barrier carbon nanotube transistor: compact modeling, scaling study, and circuit design applications. IEEE Trans Electron Devices 2011;58(1):195-205.

[29] Nojeh A. Carbon nanotube photothermionics: toward laser-pointer-driven cathodes for simple free-electron devices and systems. MRS Bull 2017;42(7):500-4.

[30] Farhana Soheli, Alam AHMZahirul, Khan Sheroz, Motakabber SMA. NEGF-based transport phenomena for semiconduncting CNTFET. In: Proceedings of the 5th national symposium on information technology: towards new smart world (NSITNSW); 2015. p. 1-3. 〈http://dx.doi.org/10.1109/NSITNSW.2015.7176386〉. 
[31] Dokania Vishesh, Islam Aminul, Dixit Vivek, Tiwari Shree Prakash. Analytical modeling of wrap-gate carbon nanotube FET with parasitic capacitances and density of states. IEEE Trans Electron Devices 2016:1-6. http://dx.doi.org/10.1109/ TED.2016.2581119.

[32] Tarfaoui M, El Moumen A, Lafdi K. Progressive damage modeling in carbon fibers/ carbon nanotubes reinforced polymer composites. Compos Part B: Eng 2017;112:185-95. [Mar 1].

[33] Rakibul Karim Akanda Md, Khosru Quazi DM. FEM model of wraparound CNTFET With multi-CNT and its capacitance modeling. IEEE Trans Electron Dev 2013;60(1):97-102.

[34] 〈https://nano.stanford.edu/stanford-cnfet-model〉.

[35] Rouf NirjhorTahmidur, Deep AshfaqulHaq, Hassan RusafaBinte, Khan SabbirAhmed, Hasan Mahmudul, Mominuzzaman SharifMohammad. Current-voltage characteristics CNTFET considering non-ballistic conduction: effect of gate oxide thickness. In: Proceedings of the 2nd international conference on devices, circuits and systems (ICDCS), IEEE 978-1-4799-1356-5/14; 2014. p. 1-4.

[36] Rouf NirjhorTahmidur, Deep AshfaqulHaq, Hassan RusafaBinte, Khan SabbirAhmed, Hasan Mahmudul, Mominuzzaman SharifMohammad. Current-voltage characteristics of CNTFET considering non-ballistic conduction: effect of dielectric constant. In: Proceedings of the 9th IEEE international conference on nano/ micro engineered and molecular systems. April 13-16, Hawaii, USA; 2014. p. $256-9$.

[37] Shafizadeh M, Rezai A. Improved device performance in a CNTFET using La_ $\{2\} O_{-}\{3\}$ high-/kappa dielectrics. J Comput Electron 2017;16(2):221-7. [Jun 1]. 